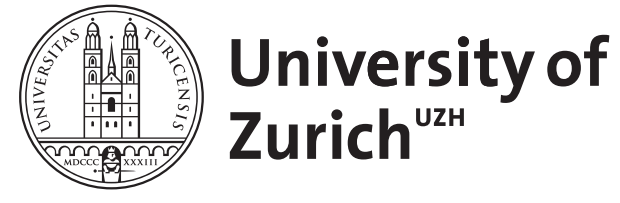

Zurich Open Repository and Archive

University of Zurich

University Library

Strickhofstrasse 39

CH-8057 Zurich

www.zora.uzh.ch

Year: 2011

\title{
Calibration protocols for fluorescent calcium indicators
}

Helmchen, F

DOI: https://doi.org/10.1101/pdb.prot5649

Posted at the Zurich Open Repository and Archive, University of Zurich

ZORA URL: https://doi.org/10.5167/uzh-54905

Journal Article

Published Version

Originally published at:

Helmchen, F (2011). Calibration protocols for fluorescent calcium indicators. Cold Spring Harbor Protocols, 2011(8):980-984.

DOI: https://doi.org/10.1101/pdb.prot5649 


\title{
Protocol
}

\section{Calibration Protocols for Fluorescent Calcium Indicators}

\author{
Fritjof Helmchen
}

\section{INTRODUCTION}

Fluorescent calcium indicators are useful for measuring intracellular calcium ion concentrations. For a quantitative understanding of the physiological roles of $\mathrm{Ca}^{2+}$, fluorescence signals measured with calcium indicators have to be converted to intracellular free calcium concentration $\left(\left[\mathrm{Ca}^{2+}\right]_{\mathrm{i}}\right)$. Most methods for converting a fluorescence signal to $\left[\mathrm{Ca}^{2+}\right]_{\mathrm{i}}$ require the determination of a set of three calibration parameters: $\left(K_{\mathrm{eff}}, R_{\min }, R_{\max }\right),\left(K_{\mathrm{d}}, \Delta F / F_{\max }\left[\mathrm{Ca}^{2+}\right]_{\mathrm{rest}}\right)$, or $\left(K_{d}, R_{\mathrm{f}}, F_{\max }\right)$ or $\left(K_{\mathrm{app}}, \tau_{\min ,}, \tau_{\max }\right)$. Here we describe the classical procedure for calibration of ratiometric measurements for both in vivo and in vitro calibrations, which is also useful for determining $K_{\mathrm{d}}$ and $R_{\mathrm{f}}$. The $\left[\mathrm{Ca}^{2+}\right]_{\mathrm{i}}$ dependence of the fluorescence ratio is measured using a set of at least three calibration solutions with known $\left[\mathrm{Ca}^{2+}\right]_{\mathrm{i}}$ levels.

\section{RELATED INFORMATION}

A detailed discussion of the theoretical background and the various principal methods for the calibration of calcium imaging data can be found in Calibration of Fluorescent Calcium Indicators (Helmchen 2011). The article also defines all the parameters and variables mentioned in this protocol and provides equations (some of them used in this protocol) which delineate the relationships between these variables.

\section{MATERIALS}

It is essential that you consult the appropriate Material Safety Data Sheets and your institution's Environmental Health and Safety Office for proper handling of equipment and hazardous materials used in this protocol.

\section{Reagents}

Calcium solutions:

- "Zero calcium" solution: Intracellular solution containing $\geq 10 \mathrm{mM} \mathrm{K}_{2} \mathrm{EGTA}$ and a low concentration $(0.1 \mathrm{~mm})$ of calcium indicator.

- "High calcium" solution: Intracellular solution containing $\geq 20 \mathrm{mM} \mathrm{CaCl}$ and $0.1 \mathrm{mM}$ calcium indicator.

- "Intermediate calcium" solutions: A mixture of two intracellular solutions containing CaEGTA and $\mathrm{K}_{2}$ EGTA. Mixed at concentrations in the $5-20-\mathrm{mM}$ range at different ratios $(1: 2,1: 1$, or $2: 1)$, these solutions can adjust (buffer) the $\left[\mathrm{Ca}^{2+}\right]_{\mathrm{i}}$ level to value(s) around the $K_{\mathrm{d}}$ of the indicator. Add a low concentration $(0.1 \mathrm{mM})$ of calcium indicator. These mixture solutions are critical as the $\left[\mathrm{Ca}^{2+}\right]_{i}$ level reached directly depends on the assumed $K_{d}$ of EGTA. Because of its lower $\mathrm{pH}$ dependence, it may be preferable to use BAPTA instead of EGTA.

Calcium calibration buffer kits for preparation of such standard calibration solutions are available from Molecular Probes (Invitrogen). Note, however, that dissociation constants of $\mathrm{Ca}^{2+}$ buffers as well as other indicator properties depend on temperature, $\mathrm{pH}$, and the ionic strength of the solution (Groden et al. 1991). Therefore, it may be sensible to prepare custom buffered solutions based on the specific intracellular solution used in order to mimic experimental

Adapted from Imaging: A Laboratory Manual (ed. Yuste).

CSHL Press, Cold Spring Harbor, NY, USA, 2010.

Cite as: Cold Spring Harb Protoc; 2011; doi:10.1101/pdb.prot5649

www.cshprotocols.org 
conditions as closely as possible. For further guidelines on the preparation of calibration solutions, see Tsien and Pozzan (1989).

Cells under study

\section{Equipment}

Beads, fluorescent, 4.5- $\mu$ m (Polysciences, Inc.) (see Step 13)

Capillaries (glass) or microslides

Equipment for whole-cell recording (see Step 7)

Microscope, fluorescence

Silicone grease

\section{METHOD}

For in vitro calibration of $\left[\mathrm{Ca}^{2+}\right]_{i}$, follow Steps $1-5$. For in vivo calibration of $\left[\mathrm{Ca}^{2+}\right]_{i}$, follow Steps 6-12. For calibration of absolute fluorescence intensity, follow Step 13.

\section{In Vitro Calibration of $\left[\mathrm{Ca}^{2+}\right]_{i}$}

1. Fill glass capillaries or microslides with the calcium calibration solutions. For measurements under a water-immersion objective, seal the ends of the microslides (e.g., with silicone grease).

2. Perform fluorescence measurements under conditions as close as possible to the experimental conditions, that is, using the same microscope objective, filter sets, and detector settings as in the imaging experiments. Measure fluorescence at both excitation wavelengths for each calibration solution.

3. In addition, measure background fluorescence values at each wavelength using capillaries filled with solution containing no dye.

4. Subtract background fluorescence values and calculate fluorescence ratios $R$ for each $\left[\mathrm{Ca}^{2+}\right]_{\mathrm{i}}$ level.

5. Calculate the three parameters $\left(K_{\mathrm{eff}}, R_{\mathrm{min}}, R_{\mathrm{max}}\right)$ from the set of calibration measurements using Equation 11 in Calibration of Fluorescent Calcium Indicators (Helmchen 2011). If more than one intermediate calcium solution has been used, obtain parameters from a fit of the data set with Equation 11.

See Troubleshooting.

In Vivo Calibration of $\left[\mathrm{Ca}^{2+}\right]_{\mathrm{i}}$

In this case, the cells under investigation are filled directly with the buffered [Ca2+]i solutions. For example, patch-clamp experiments on cultured cells or cells in brain slices provide direct access to the cytosol (Neher 1989).

6. Start with $R_{\min }$. Fill a patch pipette with the "zero calcium" calibration solution.

7. Obtain a whole-cell recording from the cell type under investigation. Use the same experimental settings as in the experiments.

8. After diffusional equilibration, measure fluorescence at both excitation wavelengths in a region of interest on the filled cell.

See Troubleshooting.

9. Measure appropriate background fluorescence values, for example, from a region next to the filled cell.

10. Subtract background fluorescence values and calculate the fluorescence ratio $R_{\min }$.

11. Repeat the measurement for at least three cells and average data.

12. Proceed in the same way (Steps 6-11) for determining $R_{\max }$ and $K_{\text {eff. }}$ 


\section{Calibration of Absolute Fluorescence Intensity}

13. In the case of calcium flux measurements, the calibration consists in the determination of the proportionality constant $f_{\max }$ (see Equation 13 in Calibration of Fluorescent Calcium Indicators [Helmchen 2011]). $f_{\max }$ may be inferred from electrical recordings of pure calcium currents and simultaneous measurements of the evoked fluorescence changes (Helmchen et al. 1997). Once $f_{\max }$ is known, changes in absolute fluorescence can be directly converted to calcium charge. To account for long-term changes in the illumination intensity or the detection efficiency in the course of these experiments, absolute fluorescence intensities should be normalized to a fluorescent standard such as $4.5-\mu \mathrm{m}$ fluorescent beads and thus expressed in "bead units" (BU) (Schneggenburger et al. 1993). The BU should be measured on each experimental day as the mean fluorescence of at least three beads.

\section{TROUBLESHOOTING}

Problem: The parameters obtained from in vitro calibrations may yield unreliable results because the behavior of fluorescent dyes is altered in the viscous cytosolic environment and by intracellular binding or uptake (Moore et al. 1990).

[Step 5]

Solution: Apparent negative $\left[\mathrm{Ca}^{2+}\right]_{i}$ values are a clear indication of this problem when in vitro calibration parameters are applied to fluorescence data from cells. Poenie (1990) suggested that viscosity can be corrected for by multiplying $R_{\min }$ and $R_{\max }$ by a factor of $0.7-0.85$. Whenever possible, an in vivo calibration is preferable.

Problem: Small cells such as chromaffin cells are readily filled with the calibration solutions (within minutes). For large cells with extensive neural processes; however, loading of the cell may take significantly longer. In addition, strong extrusion mechanisms may prevent $\left[\mathrm{Ca}^{2+}\right]_{i}$ from reaching and maintaining the concentration level in the pipette solution.

[Step 8]

Solution: In these cases, calibration fluorescence measurements should be performed very close to the pipette tip and with low access resistance (Eilers et al. 1995). In general, when using whole-cell recordings, reliable values are easily obtained for $R_{\min }$, whereas the determination of $R_{\max }$ and $K_{\text {eff }}$ is more susceptible to the problem of an insufficient clamp of $\left[\mathrm{Ca}^{2+}\right]_{i}$ to the pipette level. $R_{\max }$, however, can also be estimated at the end of or during an experiment by applying a strong stimulation (e.g., a long high-frequency train of action potentials) to saturate the indicator.

\section{DISCUSSION}

To illustrate several of the calibration techniques described above, Figure 1 shows examples of calcium measurements from large presynaptic terminals ("calyces of Held") in the medial nucleus of the trapezoid body (MNTB) (see also Helmchen et al. 1997). Using an acute brain slice preparation, nerve terminals were loaded with various indicators and using different indicator concentrations via whole-cell patch pipettes. In the left panels, the raw fluorescence data are shown, including pre- and post-stimulus measurements of the background fluorescence. These data were then evaluated as either relative fluorescence change $\Delta F / F$, fluorescence ratio, or absolute fluorescence change (middle panels). Finally, fluorescence changes were converted to calcium concentration or charge applying the set of predetermined calibration parameters.

Single-wavelength measurements using the low-affinity indicator MagFura-2 were performed to obtain an estimate of the presynaptic $\left[\mathrm{Ca}^{2+}\right]_{i}$ dynamics with minimal distortion by indicator buffering (Fig. 1A). At 380-nm wavelength, MagFura-2 fluorescence decreases upon $\mathrm{Ca}^{2+}$ binding. For calibration, a dissociation constant of $K_{\mathrm{d}}=45 \mu \mathrm{m}$ was assumed and $(-\Delta F / F)_{\max }$ was determined to be $78 \%$ using long trains of action potentials at $200 \mathrm{~Hz}$. Because a single action potential induced only a very small fluorescence change, the linearized Equation 9 (in Calibration of Fluorescent Calcium Indicators [Helmchen 2011]) could be applied, revealing a large (several hundred nanomolar) and brief (decay time constant $50 \mathrm{msec}$ ) calcium transient.

In a separate set of experiments, Fura-2 was used at a moderate concentration (160 $\mu \mathrm{M})$ and ratiometric measurements were performed using fast 360/380-nm wavelength switching with a 
A
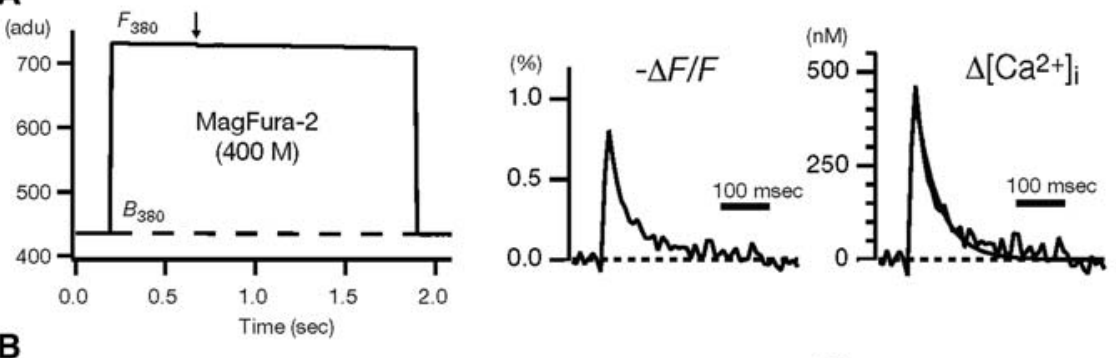

B
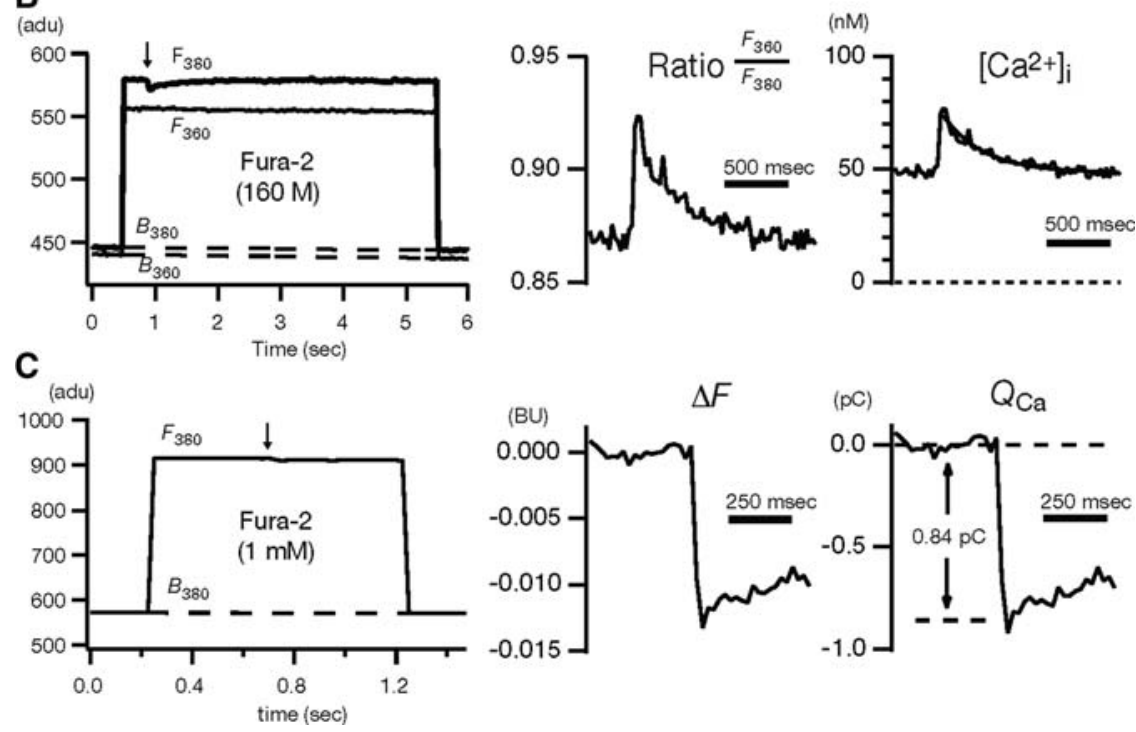

FIGURE 1. Calibration examples from measurements of single action potential-induced fluorescence changes in calyx-type presynaptic terminals in the MNTB. The fluorescence $(F)$ averaged over the entire terminal was measured using a fast chargecoupled device (CCD) camera (expressed in analog-to-digital units [adu]). Background fluorescence (B) from a nearby region was determined immediately before and after each measurement (interpolated by dashed lines). Timing of single action potentials is indicated by arrows. The temperature was $35^{\circ} \mathrm{C}$. (A) Single-wavelength measurement using the low-affinity indicator MagFura-2. A single action potential caused a small $\Delta F / F$ change of $<1 \%$, which was converted to $\Delta\left[\mathrm{Ca}^{2+}\right]_{i}$ using Equation 9 in Calibration of Fluorescent Calcium Indicators (Helmchen 2011). Average of 50 traces. (B) Ratiometric measurement using Fura-2. The ratio between the fluorescence intensities at the isosbestic wavelength $\left(F_{360}\right)$ and at 380 $\mathrm{nm}\left(F_{380}\right)$ was evaluated and converted to $\left[\mathrm{Ca}^{2+}\right]_{\mathrm{i}}$ using Equation 11 in Calibration of Fluorescent Calcium Indicators (Helmchen 2011). Note that an estimate of $\left[\mathrm{Ca}^{2+}\right]_{\text {rest }}$ is obtained, but that the $\left[\mathrm{Ca}^{2+}\right]_{i}$ transient is profoundly reduced in amplitude and prolonged owing to the added Fura-2 $\mathrm{Ca}^{2+}$-buffering capacity. No averaging. (C) Calcium flux measurement using Fura-2 overload. At high Fura-2 concentration, a single action potential induces only a small fluorescence decrement $\Delta F_{380}$ of $\sim 5$ adu $(\approx 1 \% \Delta F / F)$, which is expressed in $\mathrm{BU}$ and then converted to the total calcium charge $Q_{\mathrm{Ca}}$ using Equation 13 in Calibration of Fluorescent Calcium Indicators (Helmchen 2011). Average of 20 traces. For further details, see text.

monochromator-based illumination system (see Uhl 2010). Calibration parameters were determined using an in vivo calibration procedure by loading terminals with internal solutions clamped to zero, intermediate, and high $\left[\mathrm{Ca}^{2+}\right]_{\mathrm{i}}$, respectively (resulting in $R_{\min }=0.77 ; R_{\max }=3.15 ; K_{\mathrm{eff}}=1117 \mathrm{~nm}$ ). Note that in the case of using the isosbestic wavelength in the nominator, the $K_{\mathrm{d}}$ of the dye is given by $K_{\mathrm{d}}=K_{\text {eff }} R_{\min } /$ $R_{\max }$ yielding $K_{\mathrm{d}}=273 \mathrm{~nm}$ for Fura- 2 in our case. Conversion using these parameters yielded an estimate of $\sim 50 \mathrm{~nm}$ for $\left[\mathrm{Ca}^{2+}\right]_{\text {rest }}$. The amplitude $(26 \mathrm{nM})$ and the decay time constant (364 msec) of the single action potential-induced calcium transient were, however, clearly altered compared to the MagFura-2 measurement (Fig. 1B). This is attributed to the relatively large added $\mathrm{Ca}^{2+}$-buffering capacity compared to the endogenous $\mathrm{Ca}^{2+}$-buffering capacity (see Helmchen and Tank 2011).

Finally, Fura-2 was used at high $(1 \mathrm{~mm})$ concentration to overload the presynaptic terminal with added buffer. In this case, the change in absolute fluorescence intensity is evaluated (Fig. 1C). To normalize for changes in the imaging system over time (e.g., aging of the arc lamp), absolute fluorescence intensities were expressed in BU. The proportionality constant $f_{\max }$ was determined in separate 
experiments using pure calcium currents as $f_{\max }=0.0144 \mathrm{BU} \mathrm{pC}^{-1}$ (Helmchen et al. 1997). It was found that $\sim 1 \mathrm{pC}$ of $\mathrm{Ca}^{2+}$ enter the nerve terminal per action potential. In summary, these examples demonstrate how different methods of calcium calibration can be used to quantify various aspects of the calcium signaling system in small neuronal compartments.

\section{REFERENCES}

Eilers J, Schneggenburger R, Neher E. 1995. Patch clamp and calcium imaging in brain slices. In Single-channel recording, 2nd ed. (ed. Sakmann B, Neher E), pp. 213-229. Plenum, New York.

Groden DL, Guan Z, Stokes BT. 1991. Determination of Fura-2 dissociation constants following adjustment of the apparent Ca-EGTA association constant for temperature and ionic strength. Cell Calcium 12: 279-287.

Helmchen F. 2011. Calibration of fluorescent calcium indicators. Cold Spring Harb Protoc doi: 10.1101/pdb.top120.

Helmchen F, Tank DW. 2011. A single-compartment model of calcium dynamics in nerve terminals and dendrites. In Imaging in neuroscience: A laboratory manual (ed. Helmchen F, Konnerth A), pp. 355-368. Cold Spring Harbor Laboratory Press, Cold Spring Harbor, NY.

Helmchen F, Borst JGG, Sakmann B. 1997. Calcium dynamics associated with a single action potential in a CNS presynaptic terminal. Biophys / 72: 1458-1471.
Moore EDW, Becker PL, Fogarty KE, Williams DA, Fay FS. 1990. Ca ${ }^{2+}$ imaging in single living cells: Theoretical and practical issues. Cell Calcium 11: 157-179.

Neher E. 1989. Combined fura-2 and patch clamp measurements in rat peritoneal mast cells. In Neuromuscular junction (ed. Sellin L et al.), pp. 65-76. Elsevier, Amsterdam.

Poenie M. 1990. Alteration of intracellular Fura-2 fluorescence by viscosity: A simple correction. Cell Calcium 11: 85-91.

Schneggenburger R, Zhou Z, Konnerth A, Neher E. 1993. Fractional contribution of calcium to the cation current through glutamate receptor channels. Neuron 11: 133-143.

Tsien RY, Pozzan T. 1989. Measurement of cytosolic free $\mathrm{Ca}^{2+}$ with quin-2. Methods Enzymol 172: 256-262.

Uhl R. 2010. Arc lamps and monochromators for fluorescence microscopy. In Imaging: A laboratory manual (ed. Yuste R), pp. 117-122. Cold Spring Harbor Laboratory Press, Cold Spring Harbor, NY. 